University of Nebraska - Lincoln

DigitalCommons@University of Nebraska - Lincoln

January 2006

\title{
Cluster-Assembled Nanocomposites
}

Yinfan Xu

University of Nebraska - Lincoln, yxu2@unl.edu

M.L. Yan

University of Nebraska - Lincoln

David J. Sellmyer

University of Nebraska-Lincoln, dsellmyer@unl.edu

Follow this and additional works at: https://digitalcommons.unl.edu/physicssellmyer

Part of the Physics Commons

Xu, Yinfan; Yan, M.L.; and Sellmyer, David J., "Cluster-Assembled Nanocomposites" (2006). David Sellmyer Publications. 194.

https://digitalcommons.unl.edu/physicssellmyer/194

This Article is brought to you for free and open access by the Research Papers in Physics and Astronomy at DigitalCommons@University of Nebraska - Lincoln. It has been accepted for inclusion in David Sellmyer Publications by an authorized administrator of DigitalCommons@University of Nebraska - Lincoln. 
Published in Advanced Magnetic Nanostructures, edited by David Sellmyer and Ralph Skomski. New York: Springer, 2006. Pages 207-238. Copyright (C) 2006 Springer Science+Business Media, Inc. Used by permission.

Chapter 8

\title{
Cluster-Assembled Nanocomposites
}

\author{
Y. F. Xu, M. L. Yan and D. J. Sellmyer
}

Center for Materials Research and Analysis and

Department of Physics and Astronomy

University of Nebraska

Lincoln, NE 68588, USA

\begin{abstract}
This chapter focuses on a gas-aggregation technique to prepare magnetic nanoclusters with controllable cluster sizes and size distributions. The review includes current research on nanoclusters, such as uncoated and oxide-coated $\mathrm{Fe}, \mathrm{Co}$ and $\mathrm{Fe}$ clusters and clusters made from alloys, but special emphasis is on highly anisotropic $L 1_{0}$-ordered FePt clusters, which are of potential interest for magnetic recording with ultrahigh areal densities of more than 1 Tera bit/in ${ }^{2}$. In particular, we discuss magnetic and structural properties of FePt nanoclusters and thin films. Another approach discussed in the chapter is to create cluster nanocomposites by multilayering with post-deposition annealing. The advantage of this method is that the clusters can be oriented along a desired easy axis. One example is $L 1_{0^{-}}(001)$ oriented FePt nanocomposite films with a FePt cluster size of about $5 \mathrm{~nm}$.
\end{abstract}

\section{INTRODUCTION}

Nanostructured materials, assembled using nanoparticles or nanoclusters as building blocks, can be synthesized to have a wide variety of controlled optical, electronic, magnetic, mechanical and chemical properties with potential or realized technological applications. Simple free-metal clusters have been thoroughly investigated as reviewed by Haberland [1], W. Ekardt [2], and Meiwes-Broer [3]. In this chapter we focus on magnetic clusters and magnetic cluster-assembled nanocomposites.

In the last decade, magnetic nanoclusters with diameters of 1-10 nm and containing tens-to-thousands of atoms have been of great interest because their properties are critically dependent on size [4-10]. The total magnetic moment in free $\mathrm{Fe}, \mathrm{Co}$, and $\mathrm{Ni}$ clusters was determined as a function of size by measuring their Stern-Gerlach deflections $[4,5,9]$. For example, at a low temperature of $120 \mathrm{~K}$, for small Fe clusters $(25 \leq \mathrm{N} \leq$ 130 atoms), the moment was found to be $3 \mu_{\mathrm{B}}$ per atom, which is considerably higher than the bulk value $\left(2.2 \mu_{\mathrm{B}}\right)$; for larger Fe clusters, the mo- 
ment decreased with increasing cluster size and approached the bulk value at cluster sizes of about 500 atoms [5]. This size dependence may enable cluster-based materials to be produced with novel magnetic or electronic properties that can be used in technologies such as extremely highdensity data storage, spin electronics, memory devices, and high-performance magnetic materials.

Recently there has been considerable interest in $L 1_{0}$ ordered CoPt, Fe nanoparticles with the high magnetocrystalline anisotropy for extremely high density magnetic recording (EHDR) media [11-15]. The drive for the EHDR, i.e. from $100 \mathrm{~Gb} / \mathrm{in}^{2}$ to $1 \mathrm{~Tb} / \mathrm{in}^{2}$, imposes the need for grain size below $8 \mathrm{~nm}$ with extremely uniform grain size to maintain an optimum signal-to-noise ratio [16]. However, such small particles will cause the thermal instability of magnetic recording because of the superparamagnetic limitation. The equiatomic $L 1_{0}$ ordered phase CoPt and FePt alloys have high anisotropy constant of $\sim 5 \times 10^{7} \mathrm{erg} / \mathrm{cm}^{3}$ and $\sim 7 \times 10^{7}$ respectively [17-19], which are of crucial importance for EHDR media with a small grain size below $8 \mathrm{~nm}$, because high magnetocrystalline anisotropy is needed to create a barrier to thermally activated switching of the magnetization. Most CoPt and FePt nanoparticles were prepared with normal magnetron sputtering or electron beam evaporation techniques [1115]. However, Sun et al. prepared FePt nanoparticles with very uniform particle size and narrow size distribution by using the simultaneous reduction of platinum acetylacetonate and thermal decomposition of iron pentacarbonyl [20,21], as described in chapter 9 by S. Sun.

In this chapter, we will introduce a gas-aggregation technique, in which magnetron sputtering is employed in the source [22], for the preparation of magnetic nanoclusters and cluster-assembled nanocomposites. This cluster-deposition technique can produce a large range of mean cluster sizes, from $3 \mathrm{~nm}$ to $12 \mathrm{~nm}$, with high deposition rate (can be as high as $5 \AA / \mathrm{s}$ ), and with the cluster size and size distribution controllable. Thus it is very suitable for preparation of magnetic clusters as building blocks for nanostructured materials. A major advantage of this type of system is that the clusters have much smaller size dispersion than grains in a typical vapor-deposition system. We will first introduce the cluster sources and the gas aggregation technique, then will review elemental and alloy clusters prepared by this technique, with special emphasis on the FePt, CoPt clusters and FePt cluster-based composites. Finally, multilayers that are processed by post-deposition annealing will be described as a method for preparing oriented FePt nanocomposites.

\section{EXPERIMENT FOR CLUSTER PREPARATION}

\subsection{Cluster Source and Gas-Aggregation Technique}

Formation of metal clusters by gas aggregation, in which metal atoms are evaporated or sputtered into a cooled inert gas flow at relatively high pressure, has been well established in last decade. By repeated collisions with the carrier gas, the supersaturated metal vapor nucleates and forms clusters. The mechanism of cluster formation can be explained with homogeneous and heterogeneous nucleation theories. The gas aggregation methods have been applied extensively to produce small clusters of metals such as zinc, copper, silver etc. [23-26]. In some cases this method was used in combination with a mass filter such as a quadruple or a time-of-flight spectrometer $[27,28]$. The metal vapor for cluster source can be produced by either thermal evaporation [23-28] or sputter discharge [22,29].

The advantages of a sputtering-based cluster source are: (1) it can produce a very large range of mean cluster sizes from 200 to 15000 atoms per cluster, (2) it has a high degree of ionization from $20 \%$ to $50 \%$, depending on the target materials, and (3) a wide variety of elements and alloys can be used as source materials. Usually the deposition rate is about $3 \AA$ $\mathrm{s}$ for the total flux of the cluster beam. For charged clusters the deposition rate is about $1 \AA /$ s. Currently sputtering-based gas aggregation sources are widely used in many laboratories and are commercially available from Oxford Applied Research or Mantis Deposition [30].

Figure 1 shows a schematic drawing of a cluster-deposition system with a sputtering-based gas-aggregation source [29]. It consists of four chambers: (1) the chamber for cluster formation containing a $\mathrm{LN}_{2}$ cooled aggregation tube with a magnetron sputter discharge, (2) the deposition chamber with a substrate holder, (3) a sample load-lock chamber (not shown) for rapid substrate change, and (4) a chamber (not shown) containing the time-of-flight (TOF) mass spectrometer. Briefly, the clusters are generated in the aggregation tube, they go through two apertures B1 and $\mathrm{B} 2$, then are deposited onto the substrate $\mathrm{S}$.

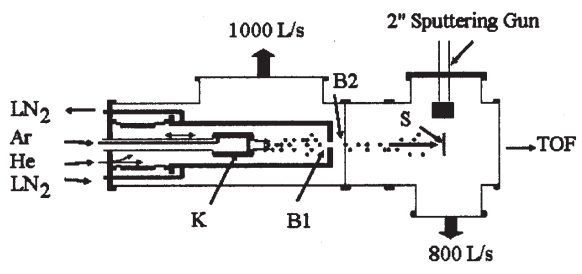

Figure 1. Schematic drawing of a cluster deposition system. 
The cluster size can be adjusted by varying the source parameters including gas-aggregation tube temperature, gas pressure, gases $\mathrm{Ar}$ to $\mathrm{He}$ flux ratio, magnetron power and the condensation length in which the clusters aggregate. The most critical variables determining the cluster size are the magnetron power and the residence time of the clusters in the aggregation region, i.e. the condensation length. Figure 2 shows a photograph of a sputtering based gas-aggregation cluster-deposition system at the University of Nebraska. The system includes a sputtering gas-aggregation source in which clusters are formed in a $0.5-1$ Torr inert gas, with diameters ranging from 3 to $12 \mathrm{~nm}$. The clusters impinge upon a substrate alone or in combination with atoms from one or two other sputtering guns. The e-beam evaporation chamber can be used to provide a thin coating (e.g. 1-3 monolayers) of oxide or other molecules. The other two sputtering guns are used to form a matrix for the magnetic clusters and/or underlayer or coating layers.

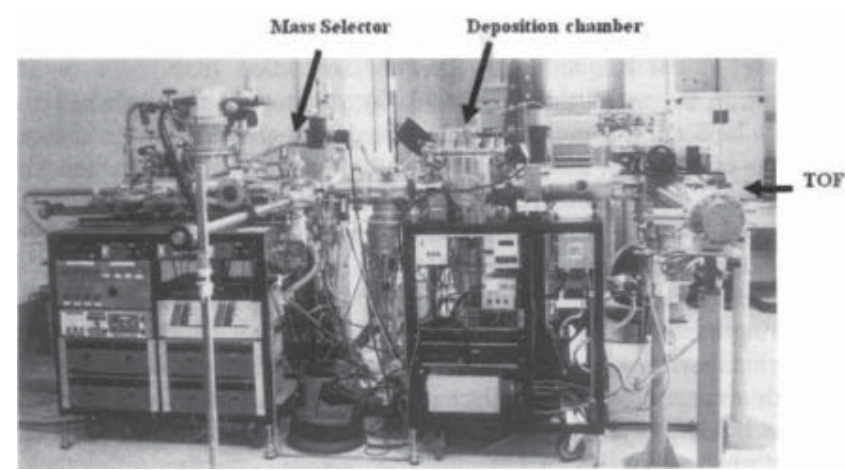

Figure 2. Cluster-deposition system at the University of Nebraska.

\subsection{Multilayer and Post-Annealing}

The non-epitaxial growth, orientated $L 1_{0}$ nanocomposite films were prepared by multilayer deposition plus post annealing. Figure 3 shows the schematic drawing of the system for preparing FePt:X $\left(=\mathrm{Ag}, \mathrm{C}, \mathrm{B}_{2} \mathrm{O}_{3}\right.$, etc.) oriented $L 1_{0}$ films. Fe/Pt/X layers were deposited individually on $\mathrm{Si}$ wafer or glass substrates by magnetron sputtering at room temperature and then annealed in vacuum for 5 to 30 minutes at various temperatures. The as-deposited multilayers were converted by phase separation into nanocomposite films with $L 1_{0}$-phase FePt grains embedded in a nonmagnetic matrix.

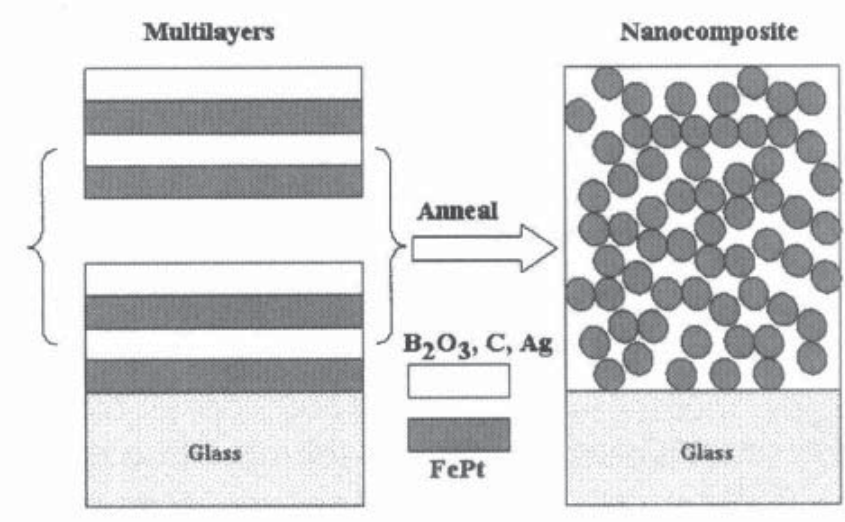

Figure 3. Preparation method of non-epitaxial growth $L 1_{0}$-phase FePt:X nanocomposite films.

\section{ELEMENTAL AND ALLOY CLUSTERS}

\subsection{Fe, Co and Ni Clusters}

Fe clusters were prepared by a gas-aggregation technique, in which metal was evaporated from a high temperature crucible to form the clusters [31]. Figure 4 is a typical STM image of Fe clusters on the Si(111) surface. In the STM images the clusters appear as isolated topographic features that are randomly distributed across the surface. The Fe cluster size fitted with a lognormal distribution, agreed with theoretical prediction for vapor growth process [32]. The effect of exposure of the Fe clusters to the atmosphere results in a significant increase in cluster size, which is consistent with total oxidation of the clusters. Magnetic circular dichroism study shows that Fe clusters with size ranging from 1 to $5 \mathrm{~nm}$ at low coverage have an orbital magnetic moment about twice that of bulk Fe [33].

The detailed magnetic behavior of $\mathrm{Fe}$ clusters has been studied for the films with Fe clusters embedded in Ag [34]. In UHV conditions, preformed Fe clusters with a mean diameter of $3 \mathrm{~nm}$ from a gas-aggregation source were deposited in conjunction with atomic Ag vapor. In such films, clusters can come into direct contact and interact via exchange. Films containing Fe cluster volume fraction from $<1 \%$ (isolated clusters) to $100 \%$ (pure clusters with no matrix) have been studied at temperatures ranging from 2 to $300 \mathrm{~K}$ by magnetometry and field-cooled (FC)/zero-field-cooled (ZFC) measurements. 


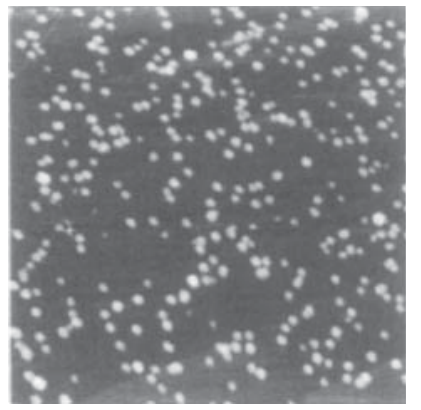

Figure 4. STM image of Fe clusters on the Si (111) surface. The image size is $200 \times 200$ $\mathrm{nm}^{2}$, after [31].

The results are summarized in the magnetic phase diagram shown in Fig. 5. At very low cluster volume fractions $(\sim 1 \%)$ and well above the cluster blocking temperature $(\sim 5 \mathrm{~K})$, the films fulfill all the conditions for ideal superparamagnetism including an $H / / T$ scaling of the magnetization curves. At $2 \mathrm{~K}$, the films demonstrate a single-particle blocking and a remanence close to $0.5 M_{\mathrm{s}}$ indicating that the particles have a uniaxial anisotropy with a random distribution of easy axis. The anisotropy constant is $2.4 \times 10^{5} \mathrm{~J} / \mathrm{m}^{3}$. At volume fractions below the percolation threshold, the high-temperature behavior can be described by interacting superparamagnetic aggregates of clusters with their moments aligned by exchange and a dipolar interaction between the aggregates. At low temperature below a collective freezing of the moments the aggregates each have a uniaxial anisotropy axis in a random direction. At high volume fraction $(>50 \%)$, above $50 \mathrm{~K}$ the magnetic configuration is a correlated superspin glass.

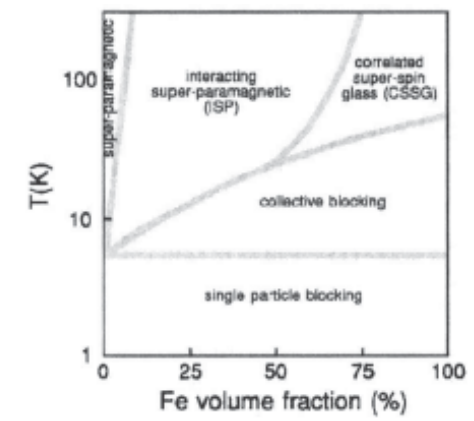

Figure 5. Magnetic phase diagram of Ag-capped Fe clusters (3 nm) in Ag matrix, after [34].
Qiang et al. studied Co clusters of a variable mean size of about 300 9000 atoms. These were produced by combining a magnetron sputtering source with a gas-aggregation tube at liquid nitrogen temperature [35]. The clusters land softly on the surface of a $\mathrm{Si}(1000)$ substrate at room temperature simultaneously along with an atomic beam of $\mathrm{Cu}$ or $\mathrm{SiO}_{2}$, which served as matrix, from a normal magnetron-sputtering gun. Figure 6(a) shows a high-resolution TEM image of a Co cluster (9000 atoms, $\mathrm{d}=6.2 \mathrm{~nm}$ ) at $10 \%$ concentration in $\mathrm{Cu}$. It can be seen that there is some interface diffusion around the Co cluster. Figure 6(b) is a nanodiffraction from the Co cluster that shows a typical $f c c$ structure. Magnetization per unit volume of $\mathrm{Co}\left(\mathrm{M}_{\mathrm{s}}\right)$ increases as one goes from (300-1000)-atom clusters to (4500-9000)-atom cluster. The 9000-atom clusters have higher $\mathrm{M}_{\mathrm{s}}$ in an insulating matrix $\left(\mathrm{SiO}_{2}\right)$ than in a metallic matrix $(\mathrm{Cu})$. Also, the $\mathrm{M}_{\mathrm{s}}$ decreases with increasing Co concentration for all cluster size, and is always lower than bulk Co value.

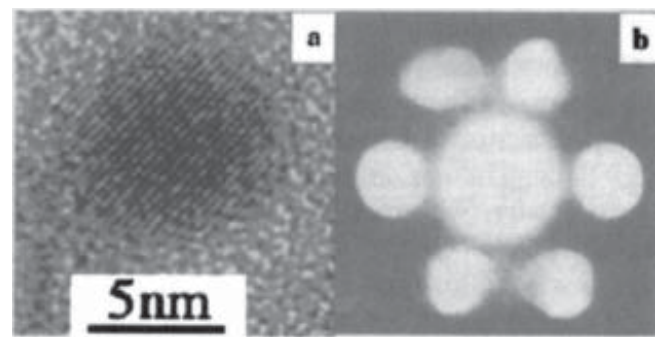

Figure 6. Co cluster having a diameter of $6.2 \mathrm{~nm}$ : (a) HRTEM image and (b) nanodiffraction pattern with a [110] zone axis.

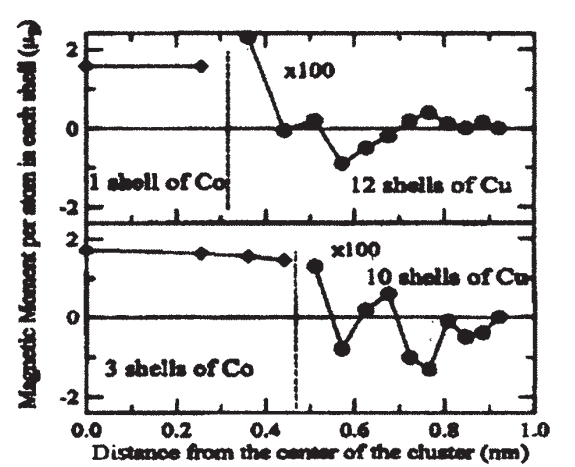

Figure 7. Calculated magnetic moments per $\mathrm{Co}$ (diamond) and $\mathrm{Cu}$ atoms (circle) as a function of the distance for one- and three-shell Co clusters. 
First-principle self-consistent electronic-structure calculations have been carried out to analyze the observed magnetization of Co clusters as a function of their size and concentration. These calculations along with a plausible cluster-matrix interface model give magnetization in good agreement with the data for low Co concentration. The moment per atom as a function of the distance from the center of the cluster is shown in Fig. 7 for clusters with 1 and 3 Co shells surrounding the central Co atom. All finitesize clusters have Co magnetic moment comparable to the bulk Co value. The neighboring $\mathrm{Cu}$ shells develop very small oscillatory magnetic moments making negligible contribution to the overall magnetization [35].

Formation of Ni clusters by gas aggregation technique was reported by Hihara et al. [36]. The cluster growth models were discussed but there are no magnetic data for Ni clusters yet.

\subsection{Oxide Coated Fe, Co Clusters}

CoO-coated Co cluster and oxide-coated Fe cluster assemblies were prepared by a plasma-gas-aggregation cluster-beam-deposition technique [37-39]. For preparation of CoO-coated Co cluster assembly, oxygen gas was introduced through a nozzle near the skimmer into the deposition chamber. The Co clusters with $\mathrm{CoO}$ shells were formed before deposition onto the substrate [37]. Figure 8 shows a TEM image of the clusters produced at oxygen gas flow rate $R\left(\mathrm{O}_{2}\right)=1 \mathrm{sccm}$. Clusters are almost monodispersed, with the mean diameter of about $13 \mathrm{~nm}$. Electron diffraction pattern indicated the coexistence of $\mathrm{Co}$ and $\mathrm{CoO}$ phases. The cluster assemblies were formed on a polyimide film with a thickness of about 100 nm.

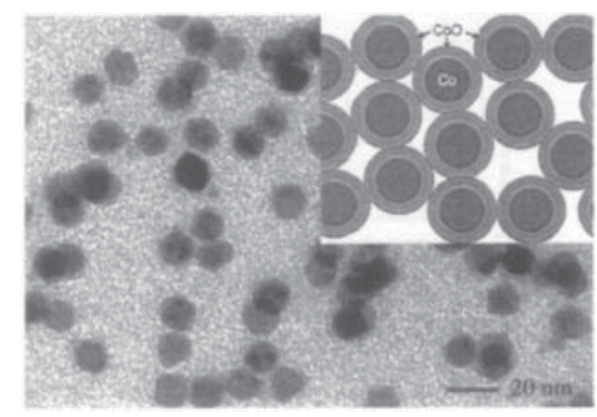

Figure 8. TEM image of CoO-coated Co clusters. The inset is a schematic drawing of the assembly of Co cores with $\mathrm{CoO}$ shells, after [37].
In order to examine the exchange coupling arising from the presence of the $\mathrm{CoO}$ phase, the hysteresis loops of CoO-coated Co cluster assemblies were measured at a temperature of $5 \mathrm{~K}$ for both zero-field-cooled (ZFC) and field-cooled (FC) samples. The large loop shift against the direction of the cooling field is detected in the FC sample, in contrast to the symmetric feature of the ZFC sample [37]. This conforms the presence of the unidirectional exchange anisotropy due to the strong exchange coupling between the ferromagnetic Co core and the antiferromagnetic $\mathrm{CoO}$ shell.

The electrical conductivity $\sigma$, and tunnel-type magnetoresistance (TMR) were measured using a conventional four-probe method. For oxygen gas flow rate $R\left(\mathrm{O}_{2}\right)<0.24 \mathrm{sccm}$, the resistivity revealed a minimum and showed a "ln $T$ " dependence at low temperature, probably due to the weak localization of conduction electrons owing to the presence of thin oxide shell covering Co cores. A small negative MR was observed in this regime. For the $R\left(\mathrm{O}_{2}\right)>0.3 \mathrm{sccm}$, tunnel-type temperature dependence of $\sigma$ in the form of "ln $\sigma$ vs. $1 / T$ " was observed between the temperatures 7 and $80 \mathrm{~K}$. This differs from the well-known temperature dependence of "In $\sigma$ vs. $1 / T^{1 / 2}$ " for disordered granular materials. The MR ratio is negative and its absolute value increases sharply with decreasing temperature below $25 \mathrm{~K}$ : from $3.5 \%$ at $25 \mathrm{~K}$ to $20.5 \%$ at $4.2 \mathrm{~K}$. This increase, by a factor of 6 , is much larger than those observed for conventional metalinsulator granular systems. These results are ascribed to a prominent cotunneling effect in the Coulomb blockade regime, arising from the uniform $\mathrm{Co}$ core size and $\mathrm{CoO}$ shell thickness in the present monodispersed cluster assemblies.

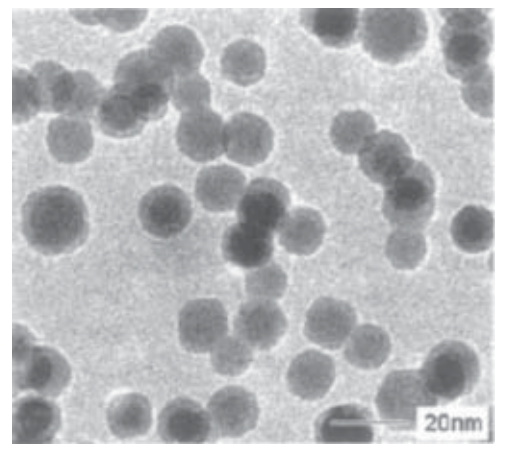

Figure 9. TEM image of the oxide-coated Fe clusters with mean diameter of $13 \mathrm{~nm}$, after [39]. 
The oxide-coated Fe cluster assembly was prepared in a similar way as the above $\mathrm{CoO}-$ coated $\mathrm{Co}$ clusters [39]. Figure 9 shows a TEM micrograph of the oxide-coated Fe clusters with a mean diameter of $13 \mathrm{~nm}$, prepared at oxygen gas flow rate of $3 \mathrm{sccm}$. Almost all clusters are characterized with a strong contrast in their "core" with a uniform gray contrast in their "shell". Electron diffraction pattern revealed that $\alpha$-Fe phase, $\mathrm{Fe}_{3} \mathrm{O}_{4}$, or $\gamma-\mathrm{Fe}_{2} \mathrm{O}_{3}$ phase are coexisting, and confirmed the core-shell structure. Here the diffraction ring for $\mathrm{Fe}_{3} \mathrm{O}_{4}$ and $\gamma-\mathrm{Fe}_{2} \mathrm{O}_{3}$ overlapped, so it is not possible to distinguish them by the electron diffraction.

The coercivity and exchange bias of the oxide-coated Fe clusters strongly depend on the cluster size, temperature and the oxygen gas flow rate, i.e., the surface oxidization degree of the clusters. For the cluster size of $9 \mathrm{~nm}$, the coercivity at $5 \mathrm{~K}$ (in both ZFC and FC cases) and the loop shift increase with the increasing oxygen gas flow rate; at room temperature, however, the coercivity becomes smaller with introduced $\mathrm{O}_{2}$ gas. The $H_{c}$ versus $T^{1 / 2}$ fitting gives the effective anisotropy constant Keff values of $3.09 \times 10^{6}, 4.29$ $\times 10^{6}$, and $5.63 \times 10^{6} \mathrm{erg} / \mathrm{cm}^{3}$ for oxygen gas flow rate of 0,1 , and $3 \mathrm{sccm}$, respectively. The onset of loop shift and bifurcation between $H_{c}(\mathrm{ZFC})$ and $H_{c}(\mathrm{FC})$ below about $T=50 \mathrm{~K}$ can be ascribed to an exchange interaction between the ferromagnetic Fe core and the freezing layers of disordered surface spins of the oxide shell crystallites, which is also the predominant origin of an enhancement of the magnetic anisotropy at low temperatures.

\subsection{Alloy Cluster Formation in Co-Al, Co-Pt Systems}

From the viewpoint of application, formation of stable alloy clusters with intermetallic compound structure is highly desired. So far the formation of ordered intermetallic alloy clusters is quite limited. Although the sputtering source can generate almost any kind of metal vapors which makes the gas- aggregation technique very promising, the synthesis of alloy clusters that possess an ordered structure is quite challenging. Konno et al. studied the alloy cluster formation in CoAl and CoPt systems [40, 41]. CoAl and CoPt were chosen because of the well-known stability of the ordered B2 (CsCl-type) structure for the former and $L 1_{0}$ (AuCu-type) structure for the latter near the composition range of $\mathrm{Co}: \mathrm{M}=1: 1(\mathrm{M}=\mathrm{Al}$ or $\mathrm{Pt})$. Furthermore, the free energy change, i.e. the heat of formation for CoAl phase (B2, $-60 \mathrm{~kJ} / \mathrm{mol})$ is much larger than that of $\mathrm{CoPt}\left(L 1_{0},-14 \mathrm{~kJ} / \mathrm{mol}\right)$. They found that $\mathrm{B} 2-\mathrm{CoAl}$ clusters were formed for a composition range wider than that predicted by the $\mathrm{Co}-\mathrm{Al}$ phase diagram, and that high-temperature $f c c$-CoPt clusters were formed in the CoPt system.
Compared with the heat of formation of $L 1_{0} \mathrm{CoPt}$, another important $L 1_{0}$ phase $\mathrm{FePt}$ has a larger value $(-25 \mathrm{~kJ} / \mathrm{mol})$ [42]. The ordering temperature for the latter is about $100{ }^{\circ} \mathrm{C}$ lower than that of the former. This result may provide a hint: large heat of formation may be favorable for the formation of ordered alloy clusters in the as-deposited state.

\section{4. $L 1_{0}$ FePt, CoPt NANOCLUSTERS}

\subsection{Size Distribution and Nanostructure of Clusters}

For free-clusters, the cluster size distribution can be measured by the time-of-flight mass spectrometer; for cluster films deposited on substrate by the cluster beam, the measurement of size distribution and observation of nanostructure are mostly done using transmission electron microscopy (TEM). In this section we will focus on the latter and pay special attention to FePt, CoPt clusters which have high anisotropy $L 1_{0}$ phase after annealing [43-45]. For the TEM observations, FePt, CoPt nanoclusters, produced in a gas-aggregation chamber, in which high pressure $\mathrm{Ar}$ gas $(\sim$ 0.5-1 Torr) was applied and cooled by $\mathrm{LN}_{2}$, were directly deposited onto carbon-coated films supported by $\mathrm{Cu}$ grids.

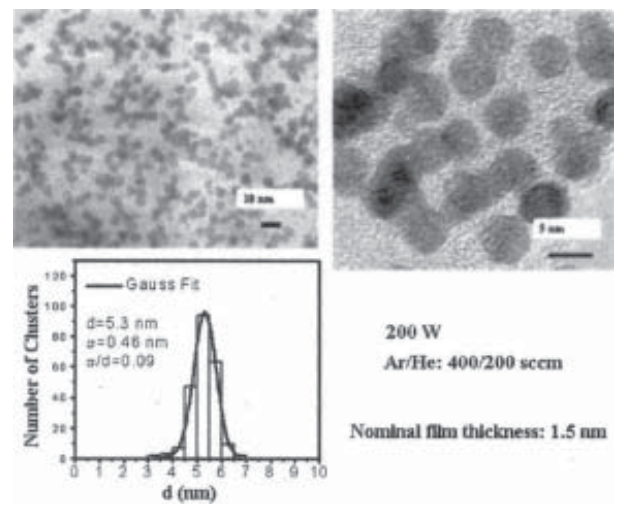

Figure 10. TEM images of FePt clusters with cluster size distribution. Right: larger magnification of the clusters.

The cluster size was controlled in a range from $3 \mathrm{~nm}$ to $6 \mathrm{~nm}$ by adjusting the preparation parameters such as sputtering power, Ar-He gases flow and gas pressure etc. Uniform cluster size distribution with standard deviation $\sigma / \mathrm{d}<0.1$ is obtained. The size distribution can be further improved if desired by applying a mass selector. Figure 10 shows the TEM 
image of as-deposited FePt nanoclusters. The corresponding cluster size distribution counted by more than 200 clusters from lower magnification TEM pictures is also included. Spherical clusters with an average size of $5.3 \mathrm{~nm}$ fitted with Gaussian distribution (with standard deviation $\sigma=0.46$ $\mathrm{nm}, \sigma / \mathrm{d}=0.09$ ) were obtained with sputtering power of $200 \mathrm{~W}$ and $\mathrm{Ar} / \mathrm{He}$ gas flow of $400 / 200 \mathrm{sccm}$.

High resolution TEM image reveals some faceting of the FePt clusters as shown in Fig. 11. An enlarged FePt cluster image shows the lattice fringes, indicating the single-crystal structure.

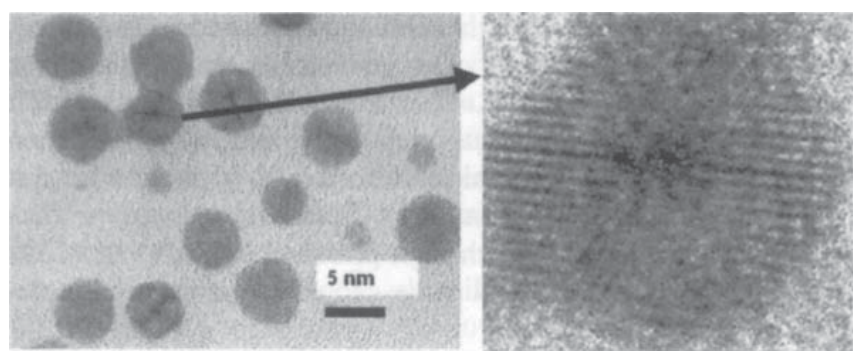

Figure 11. HRTEM images of FePt clusters with facet feature. Right: an enlarged cluster showing lattice fringe.

Similar results were also obtained for CoPt nanoclusters and shown in Fig. 12 is the high resolution TEM image for CoPt clusters with average size of $4.9 \mathrm{~nm}(\sigma=0.46 \mathrm{~nm}, \sigma / \mathrm{d}=0.09)$, prepared with sputtering power of $160 \mathrm{~W}$.

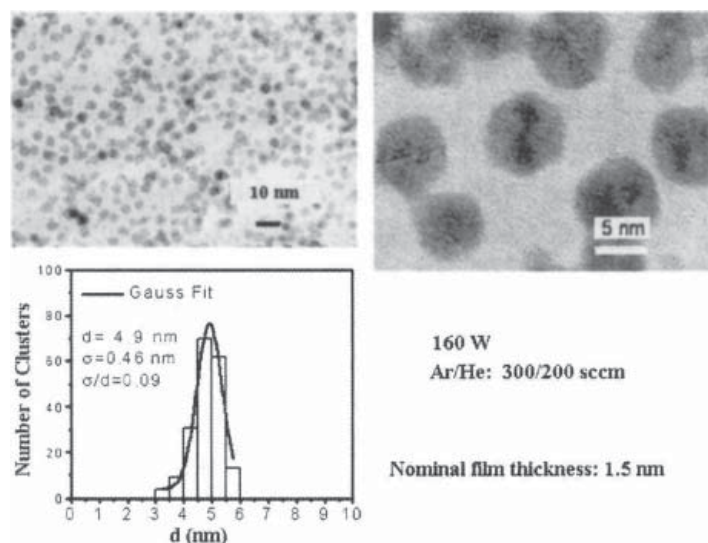

Figure 12. TEM images of CoPt clusters with cluster size distribution. Right: larger magnification of the clusters.
Figure 13 shows the sputtering power dependence of cluster size. It can be seen that FePt, CoPt cluster size has a nearly linear relationship with the sputtering power of the cluster source. It is noted that the cluster size follows a Gaussian distribution rather than a lognormal one as observed in Fe clusters [31]. This may be related to the cluster formation mechanism involved in the present method, which is different from the mechanisms for lognormal distribution. A similar Gaussian distribution was also reported in CoAl clusters [40].

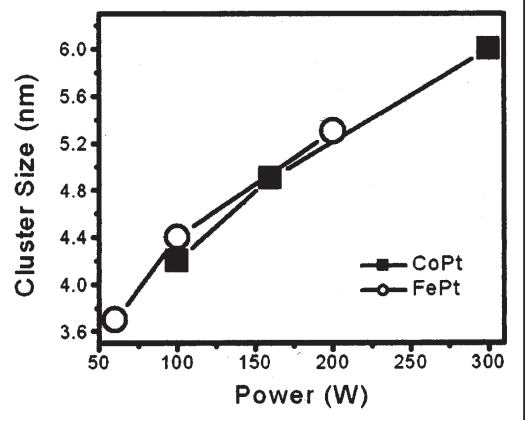

Figure 13. FePt, CoPt cluster size versus sputtering power.

In order to obtain $L 1_{0}$ ordered FePt clusters, high temperature post-deposition annealing is usually required. However, post-deposition annealing leads to subsequent crystallite growth and concurrent agglomeration of the clusters on the substrate. Rellinghaus et al. employed a sintering oven between the cluster source and deposition chamber, allowing preparation and thermal sintering of FePt nanoclusters in the gas-phase prior to their deposition $[46,47]$. The sintering temperature of the oven can be varied in the range from 293 to $1273 \mathrm{~K}$; the residence time of the clusters within the oven is between $0.05 \mathrm{~s}$ and $0.9 \mathrm{~s}$, depending on the gas flow rate in the oven. High resolution TEM observation reveals that the spherical shaped clusters are of icosahedral symmetry when prepared at gas pressure of 0.4 Torr and sintering temperature up to $1273 \mathrm{~K}$, and the relative amount of icosahedral clusters increases with increasing sintering temperature as $T_{\mathrm{s}} \geq 800 \mathrm{~K}$. Figure 14 shows a HRTEM image of an FePt icosahedron, prepared at gas pressure of about 0.4 Torr and sintering temperature of $1073 \mathrm{~K}$, with its 3-fold symmetry axis oriented parallel to the electron beam. For FePt clusters prepared at a pressure $>0.4$ Torr and high sintering temperature $(1273 \mathrm{~K})$, single-crystalline clusters with the $L 1_{0}$-ordered phase were observed. 


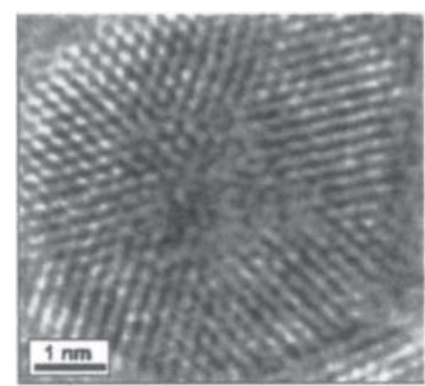

Figure 14. HRTEM image of an FePt cluster with icosahedral symmetry, after [47].

\subsection{Magnetic Properties of FePt Clusters}

Depending on the gas-pressure during preparation, the structure of the unannealed FePt clusters is icosahedral ( $\sim 0.4$ Torr) or polycrystalline $f c c$ phase ( $>0.75$ Torr ) [47]. Temperature-dependent magnetization measurements revealed that these clusters are superparamagnetic at room temperature. Clusters of icosahedral structure withstand even the highest annealing or sintering temperature $(1273 \mathrm{~K})$ and are still superparamagnetic at room temperature. Polycrystalline $f c c$ FePt nanoclusters, on the other hand, are transformed to the chemically ordered $L 1_{0}$ phase by annealing at elevated temperature. Both the blocking temperature $T_{\mathrm{b}}$ and the coercivity increased with the increase of $T_{\mathrm{s}}$. Figure 15 shows the hysteresis loops of polycrystalline FePt nanoclusters prepared at gas pressure of 1.1 Torr and sintering temperature of $1273 \mathrm{~K}$. The magnetization loops measured at temperatures between 5 and $293 \mathrm{~K}$. The coercivity increases with the decrease of temperature; a coercivity of $2.3 \mathrm{kOe}$ at temperature of $5 \mathrm{~K}$ indicated the high anisotropy $L 1_{0}$ structure, although the degree of $L 1_{0}$ ordering is not very high.

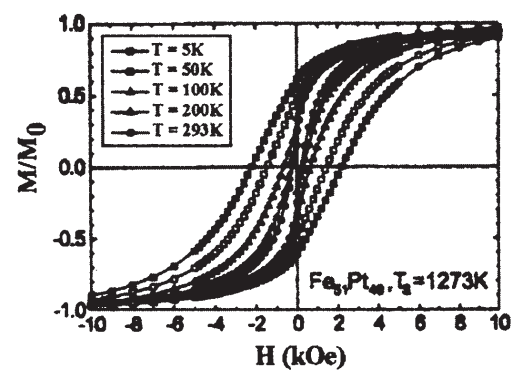

Figure 15. Hysteresis loops of polycrystalline FePt clusters sintered at $1273 \mathrm{~K}$, after [47].

\section{FePt:X (X=Ag, C) CLUSTER FILMS}

For most technological applications, the properties of clusters embedded in magnetic or non-magnetic, metallic or non-metallic matrices are more important than those of free clusters. Figure 16 shows a schematic drawing of cluster film deposition: a cluster beam combined with two atomic beams from normal magnetron sputtering guns, which are used for matrix materials deposition, such as $\mathrm{Ag}, \mathrm{C}$, etc.

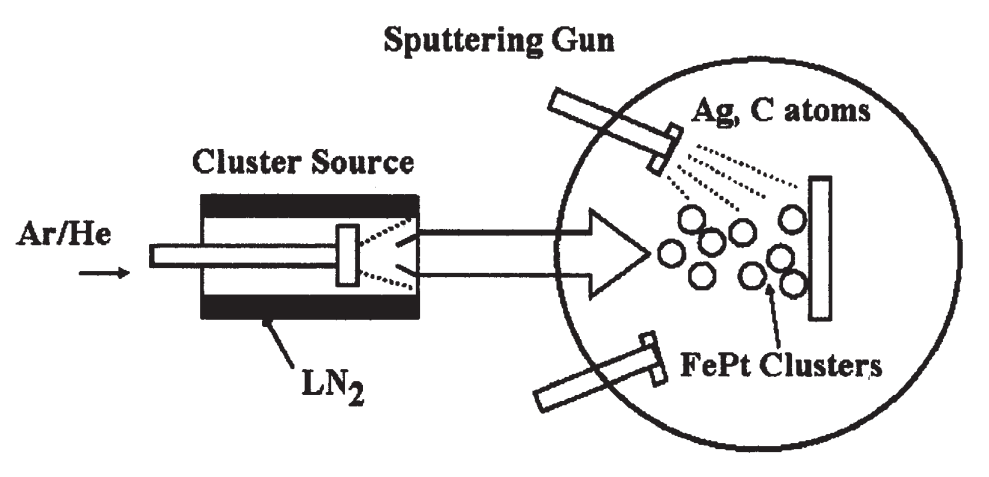

Figure 16. Schematic drawing of cluster deposition with matrix atoms beam.

Figure 17 gives two examples of cluster film structure: (a) clusters are embedded in matrix by co-deposition or, (b) isolated by a matrix via multilayering. The ability of the deposition technique to independently control these parameters makes it ideal for systematic studies of magnetic clusters. In this section, some recent work on FePt:Ag and FePt:C cluster films from our laboratory are reviewed.

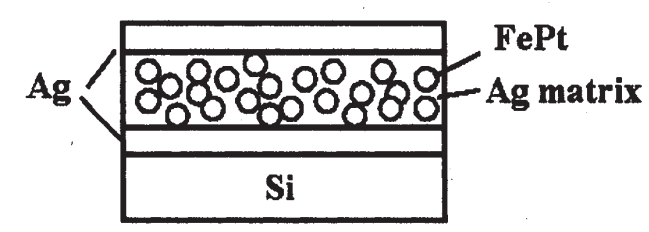

(a)

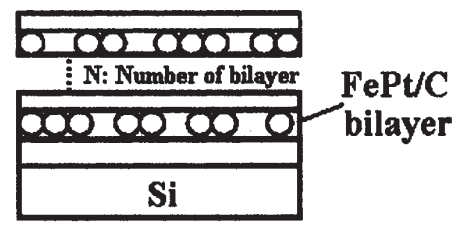

(b)
Figure 17. Cluster film structures: (a) Co-deposition and (b) multilayers. 


\subsection{FePt:Ag Cluster Films}

FePt:Ag cluster films are deposited with a three-layer structure ( $\operatorname{Ag}(2 \mathrm{~nm}) /$ $\mathrm{FePt}(12 \mathrm{~nm}) / \mathrm{Ag}(2 \mathrm{~nm})]$ or co-sputtering FePt clusters and $\mathrm{Ag}$ atoms on a $\mathrm{Si}$ substrate. The FePt clusters are produced with a power of $100 \mathrm{~W}$. The mean diameter of the clusters is about $4.4 \mathrm{~nm}$. For the three-layer films, the magnetic layer thickness is about $12 \mathrm{~nm}$, which is nearly 3 layers of FePt clusters [43]. X-ray diffraction (XRD) measurement confirmed the ordering of $L 1_{0}$ phase by post-deposition annealing. Figure 18 shows the XRD $(\theta-2 \theta)$ scans of FePt:Ag cluster film, prepared by co-sputtering, annealed at 500,600 , and $700{ }^{\circ} \mathrm{C}$ for $10 \mathrm{~min}$, respectively. The as-deposited film showed a broad FePt $f c c$ (111) peak and $\mathrm{Ag} f c c$ (111) and (200) peaks. After annealing at higher temperature, the $f_{c t}-(200)$ and (002) peaks are well-separated, and the intensity of $f c t-(001)$ peak increased with the increase of annealing temperature, indicating that the degree of $L 1_{0}$ ordering increased with the annealing temperature. In all of these films, the orientation of the $f c t \mathrm{FePt}$ clusters is random. Magnetic properties of the FePt:Ag cluster films were measured by SQUID.

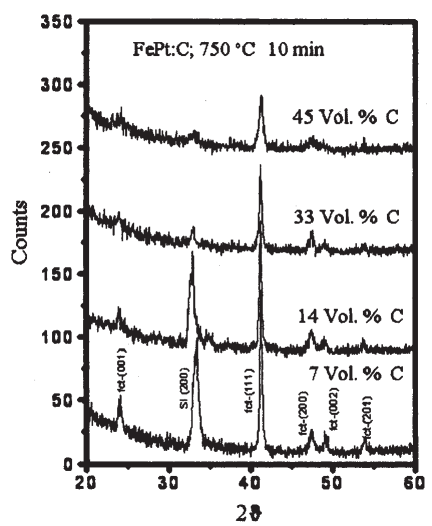

Figure 18. XRD spectra of FePt:Ag cluster films annealed at 500, 600, and $700{ }^{\circ} \mathrm{C}$ for 10 min.

Figure 19 shows the room temperature hysteresis loops of the FePt: $\mathrm{Ag}$ films annealed at 550 and $700{ }^{\circ} \mathrm{C}$ for $10 \mathrm{~min}$. Both in-plane (H // film plane) and perpendicular $(\mathrm{H} \perp$ film plane) loops are presented. The perpendicular coercivity is almost the same as the in-plane coercivity, indicating that the easy axis of clusters is distributed randomly. This result is consistent with that obtained by XRD measurement (Fig. 18).

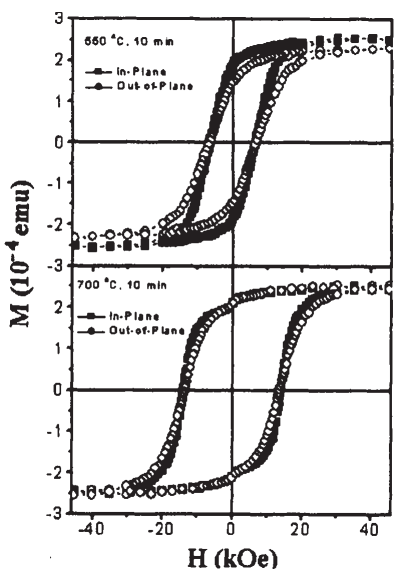

Figure 19. Hysteresis loops of FePt:Ag cluster films annealed at 550 and $700{ }^{\circ} \mathrm{C}$ for 10 min.

Figure 20 shows the dependence of the coercivity on annealing temperature for co-sputtered FePt:Ag films. The coercivity increases almost linearly with increase of annealing temperature. This can be understood through the increase of the degree of $L 1_{0}$ ordering. The degree of ordering depends on the annealing temperatures; large coercivity of the cluster films originated from the large $K_{\mathrm{u}}$ of the $f c t$ phase. Note that a coercivity more than $4 \mathrm{kOe}$ can be achieved just by annealing the film at $450{ }^{\circ} \mathrm{C}$ for $10 \mathrm{~min}$, which otherwise could be obtained by annealing samples at 550 ${ }^{\circ} \mathrm{C}$ or higher for $\mathrm{FePt}$ cluster films without $\mathrm{Ag}$ addition. This result suggests that addition of $\mathrm{Ag}$ in FePt cluster films can lower the $L 1_{0}$ ordering temperature and increase the coercivity of the cluster films significantly.

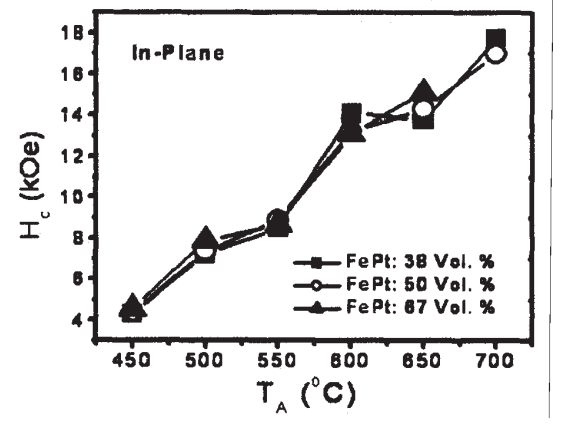

Figure 20. Effect of annealing temperature on coercivities for FePt:Ag cluster films. 


\subsection{FePt:C Cluster Films}

FePt:C nanocluster films were prepared with a multilayer method in which $\mathrm{FePt}$ cluster layers and $\mathrm{C}$ layers were alternately deposited onto a $\mathrm{Si}$ substrate. C served to isolate the clusters and to avoid cluster-aggregation during thermal annealing. The average FePt cluster size is about $4.5 \mathrm{~nm}$. The nominal thickness of each cluster layer is about $1 \mathrm{~nm}$, so that the FePt clusters can be well separated with $\mathrm{C}$ layer. The thickness of $\mathrm{C}$ layer was adjusted with $C$ volume fraction changing from 7 to $45 \%$ [48].

Figure 21 shows the TEM plan-view images for the FePt:C film with 45 vol. $\% \mathrm{C}$ annealed at $650{ }^{\circ} \mathrm{C}$ for $10 \mathrm{~min}$. It can be seen that FePt clusters are embedded in $\mathrm{C}$ matrix. Shown in Fig. 21(b) is the high resolution TEM image. It shows that single FePt clusters with diameter about $4.5 \mathrm{~nm}$ were well separated by amorphous $\mathrm{C}$ matrix; the cluster growth during high temperature annealing has been remarkably reduced with the isolation of higher volume fraction of $\mathrm{C}$.

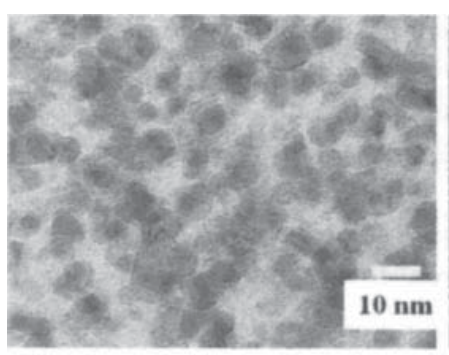

(a)

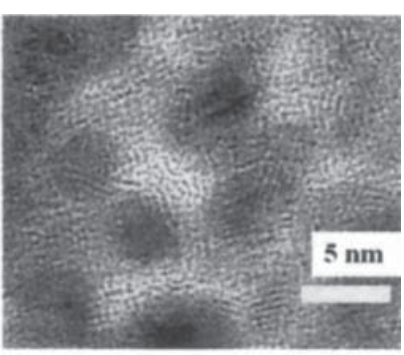

(b)
Figure 21. TEM images of FePt:C cluster film with 45 vol. $\% \mathrm{C}$, annealed at $650{ }^{\circ} \mathrm{C}$ for 10 $\min$.

XRD measurements confirmed the ordering of the $L 1_{0}$ structure [48]. The intensity of the $L 1_{0}$-peaks increases with annealing temperature, indicating the increase of the degree of $L 1_{0}$ ordering upon annealing. Figure 22 shows the effect of annealing temperature $\mathrm{T}_{\mathrm{A}}$ on the in-plane and perpendicular coercivities of FePt:C films with 45 vol. \% C. Both in-plane ( $\mathrm{H} / /$ film plane) and perpendicular $(\mathrm{H} \perp$ film plane) coercivities increased with increase of annealing temperature. The perpendicular coercivity larger than $13 \mathrm{kOe}$ was achieved in the films after annealing at a temperature of $700{ }^{\circ} \mathrm{C}$ and above. In order to determine the thermal effect on coercivity, the hysteresis loops of FePt:C cluster films were measured at low temperature down to $10 \mathrm{~K}$.

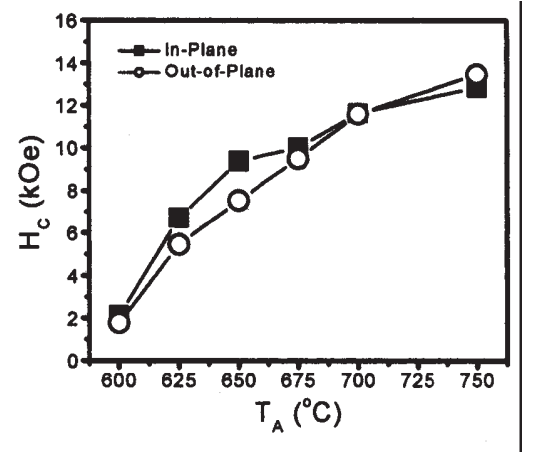

Figure 22. Effect of annealing temperature on coercivities of FePt:C cluster film with 45 vol. $\% \mathrm{C}$.

Figure 23 shows the coercivity dependence on temperature for $\mathrm{FePt}: \mathrm{C}$ cluster films with 45 vol. $\% \mathrm{C}$ from 10 to $300 \mathrm{~K}$. The films were annealed at different temperature for $10 \mathrm{~min}$. The coercivity decreases with increase of measuring temperature, for example, dropping from $19 \mathrm{kOe}$ at $10 \mathrm{~K}$ to about $13 \mathrm{kOe}$ at $300 \mathrm{~K}$ for a film annealed at $750{ }^{\circ} \mathrm{C}$ for $10 \mathrm{~min}$. This may be caused by a contribution of intrinsic temperature dependence of the anisotropy and magnetization, and thermal activation effects [49].

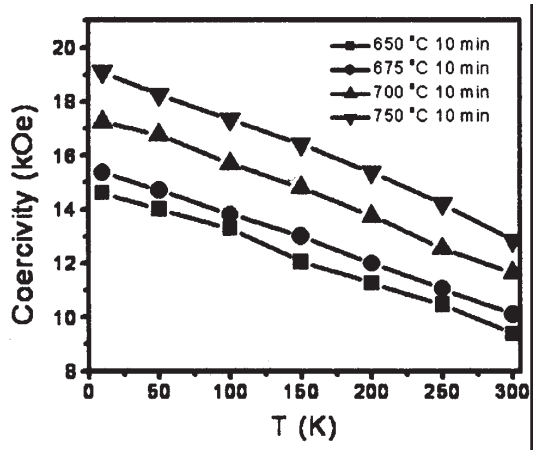

Figure 23. Temperature dependence of coercivity for FePt:C cluster films with 45 vol. \% C, annealed at temperatures from 650 to $750^{\circ} \mathrm{C}$.

Figure 24 shows the $\Delta \mathrm{m}$ curve measured for the FePt:C film with 45 vol. $\% \mathrm{C}$ annealed at $625^{\circ} \mathrm{C}$ for $10 \mathrm{~min}$ [48]. The negative value of $\Delta \mathrm{m}$ peak $(-0.17)$ suggests dipolar interactions. 


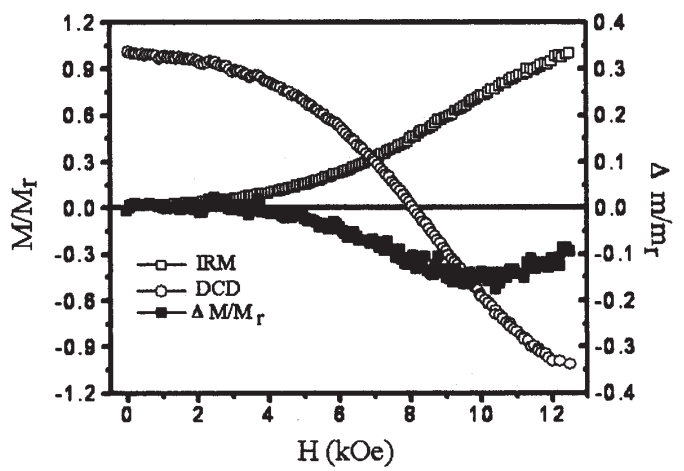

Figure 24. $\Delta \mathrm{m}$ curve for FePt:C cluster film with 45 vol. \% C annealed at $625^{\circ} \mathrm{C}$ for 10 $\min$.

\subsection{Dynamics of Magnetization Reversal in FePt:C Cluster Films}

As grain size becomes smaller and smaller with increased recording areal density, the magnetic moment in a grain may reverse spontaneously via thermal activation. Therefore, understanding the magnetization reversal behavior of media is of essential importance to control and design new media with desired properties and good thermal stability. We carried out a set of moment decay measurements for FePt:C films with 45 vol. \% C. The data were fitted with the Sharrock formula [50], and the thermal stability factor and anisotropy constant were extracted from the fitting parameters. The detailed experiment and fitting procedures can be found in Refs. [48, 51].

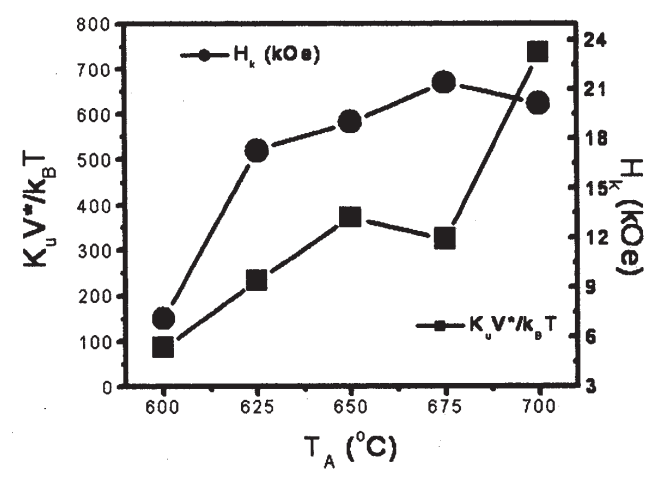

Figure 25. Effect of annealing temperature on thermal stability and magnetic anisotropy field of FePt:C cluster films with 45 vol. \% C.
Figure 25 shows the annealing temperature effect on the thermal stability factor $K_{\mathrm{u}} V^{*} / k_{\mathrm{B}} T$ [49] and anisotropy field $H_{\mathrm{k}}$ for the FePt:C films with 45 vol. $\%$ C. $H_{\mathrm{k}}$ was calculated from $H_{0}=0.48 H_{\mathrm{k}}, H_{0}$ was obtained from the Sharock fitting parameter. $H_{\mathrm{k}}$ increases rapidly with annealing temperature $T_{\mathrm{A}}$ between 600 and $625^{\circ} \mathrm{C}$, then increases slowly and to saturation for $T_{\mathrm{A}}>625^{\circ} \mathrm{C} . K_{\mathrm{u}} V^{*} / k_{\mathrm{B}} T$ increases linearly with $T_{\mathrm{A}}$ except for the point at $675{ }^{\circ} \mathrm{C}$ that might be caused by either experimental error or the activation volume $V^{*}$ being unusually small. Since Ku would be constant after the completion of $L 1_{0}$ ordering, the further increase of $K_{\mathrm{u}} V^{* /}$ $k_{\mathrm{B}} T$ with $T_{\mathrm{A}}$ is mainly due to the increase of $V^{*}$. As shown in Fig. 26, $K_{\mathrm{u}}$ is about $1.2 \times 10^{7} \mathrm{erg} / \mathrm{cm}^{3}$ for $T_{\mathrm{A}}>625^{\circ} \mathrm{C} ; V^{*}$ increases slowly initially and then increases more rapidly with $T_{\mathrm{A}}$, which results in the quasi-linear increase of $K_{\mathrm{u}} V^{*} / k_{\mathrm{B}} T$.

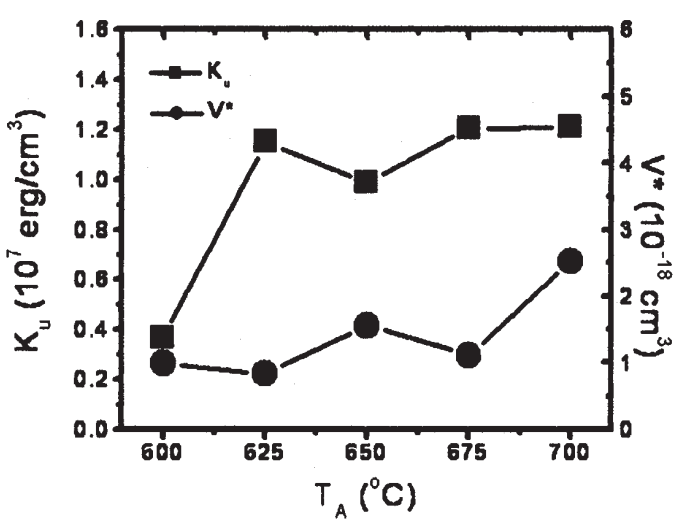

Figure 26. Effect of annealing temperature on magnetic anisotropy constant and activation volume of FePt:C cluster films with 45 vol. \% C.

\subsection{Dilute FePt:C Cluster Film: Stoner-Wohlfarth-Like Behavior}

Understanding the magnetic properties of a collection of well-isolated clusters is of great interest for exploring FePt clusters as a media for EHDR beyond 1 Tera bit/in. Dilute FePt:C cluster films were prepared by the multilayer method as described earlier. Thus Stoner-Wohlfarth-like behavior has been observed in a FePt:C cluster film with FePt volume fraction of 5\% [45]. Figure 27 shows the zero-field-cooled (ZFC) and fieldcooled (FC) magnetization curves for as-deposited and annealed FePt:C cluster film with 5 vol. \% FePt. 

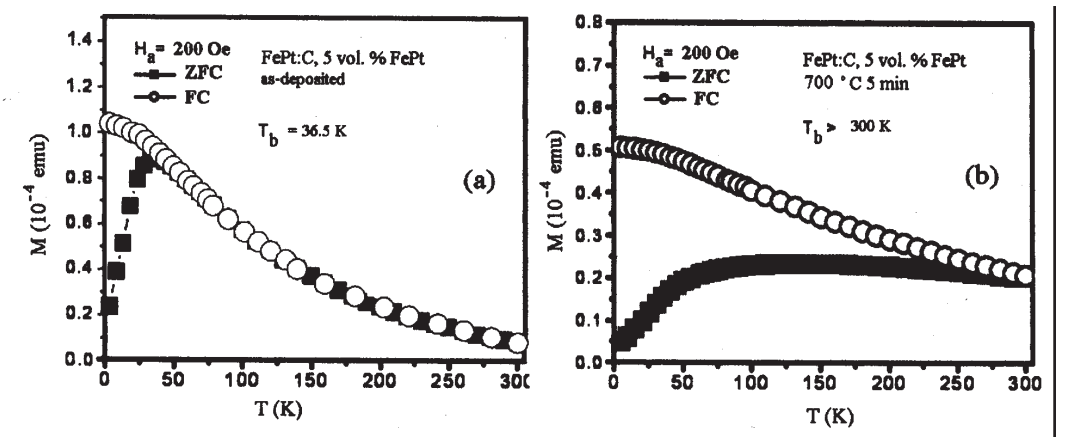

Figure 27. ZFC and FC magnetization curves of FePt:C cluster film with 5 vol. \% FePt: (a) as-deposited; (b) annealed at $700{ }^{\circ} \mathrm{C}$ for $5 \mathrm{~min}$.

An arrow in Fig. 27(a) indicates a block temperature $T_{\mathrm{b}}$ where ZFC and ZF curves are coincident. For as-deposited film, the $T_{\mathrm{b}}$ is about $36.5 \mathrm{~K}$; after annealing at $700{ }^{\circ} \mathrm{C}$ for $5 \mathrm{~min}$., the peak in $\mathrm{ZFC}$ curve was not observed, Fig. 27(b), suggesting a $T_{\mathrm{b}}$ higher than $300 \mathrm{~K}$ and the increase of anisotropy constant $\mathrm{K}_{\mathrm{u}}$ due to order or partial order of the $L 1_{0}$ phase. Figure 28 shows the temperature dependence of coercivity for FePt:C cluster films with 5 and 30 vol. $\% \mathrm{FePt}$ annealed at $700{ }^{\circ} \mathrm{C}$ for $5 \mathrm{~min}$. At room temperature, a few hundred Oe of coercivity was observed for both films; at low temperature $(10 \mathrm{~K})$, the coercivity reaches to $4 \mathrm{kOe}$ for 5 vol. $\%$ FePt film and $5.6 \mathrm{kOe}$ for 30 vol. \% FePt. Increase of the FePt cluster volume fraction increases the coercivity. These results suggest the partial ordering of $L 1_{0}$ phase at short-time annealing $(5 \mathrm{~min}$ ) and some very small clusters showing superparamagnetic behavior at room temperature.

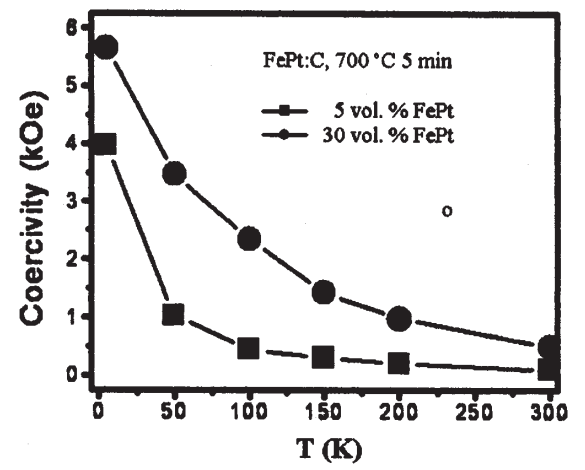

Figure 28. Temperature dependence of coercivity for dilute FePt:C cluster films annealed at $700^{\circ} \mathrm{C}$ for $5 \mathrm{~min}$
Figure 29 shows a TEM image of FePt:C cluster film with 5 vol. \% FePt annealed at $700{ }^{\circ} \mathrm{C}$ for $10 \mathrm{~min}$. Well-isolated clusters with single crystal $L 1_{0}$ structure are observed. The arrows indicate the clusters with lattice fringe observable, suggesting single crystalline clusters.

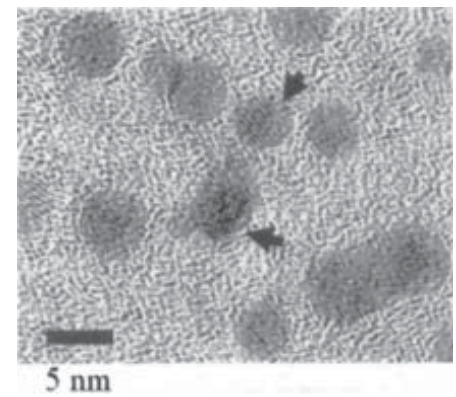

Figure 29. TEM image of FePt:C cluster film with 5 vol. $\% \mathrm{FePt}$ annealed at $700{ }^{\circ} \mathrm{C}$ for 10 min.

Shown in Fig. 30 is the temperature dependence of coercivity for the FePt:C films annealed at $700{ }^{\circ} \mathrm{C}$ for 10,30 , and $60 \mathrm{~min}$. respectively. High coercivities ( $>21 \mathrm{kOe}$ ) at room temperature were observed and they reached about $40 \mathrm{kOe}$ at low temperature. This result indicates the high degree of $L 1_{0}$ ordering after annealing for longer time (> $10 \mathrm{~min}$. ).

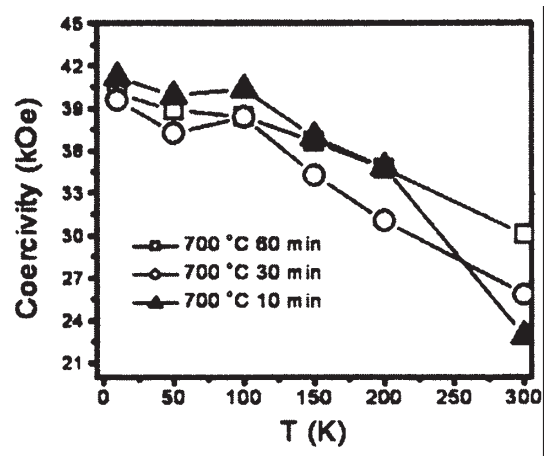

Figure 30. Temperature dependence of coercivity of dilute FePt:C cluster films with 5 vol. $\%$ FePt annealed at $700{ }^{\circ} \mathrm{C}$ for 10,30 , and $60 \mathrm{~min}$.

Fig. 31 shows the in-plane and perpendicular plane hysteresis loops of the FePt:C film with 5 vol. $\% \mathrm{FePt}$ annealed at $700{ }^{\circ} \mathrm{C}$ for $60 \mathrm{~min}$, mea- 
sured at $10 \mathrm{~K}$ with a SQUID magnetometer. A perpendicular coercivity of about $40 \mathrm{kOe}$ was achieved. The loops are not saturated under an applied field of $70 \mathrm{kOe}$. The law-of-approach to saturation was applied and the ratio of $M_{\mathrm{r}} / M_{\mathrm{s}}$ is estimated between $0.5-0.6$. Assuming these clusters are non-interacting and the formula $H_{\mathrm{c}}=0.48 H_{\mathrm{k}}$ can be applied; an anisotropy field of $83 \mathrm{kOe}$ is estimated.

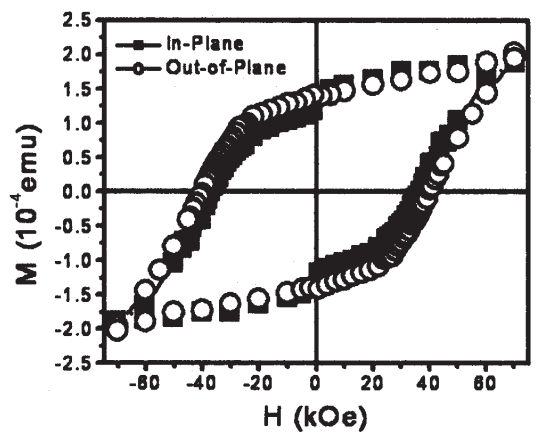

Figure 31. Hysteresis loops of FePt:C cluster film with 5 vol. \% FePt annealed at $700{ }^{\circ} \mathrm{C}$ for $60 \mathrm{~min}$, measured at $10 \mathrm{~K}$.

In summary the FePt nanoclusters studied are thermally stable, have a narrow size distribution and controllable coercivities. Their properties are similar to those required for magnetic recording at extremely high densities, i.e. approaching an areal density of $1 \mathrm{Tera} \mathrm{bit} / \mathrm{in}^{2}$. For practical application, the FePt cluster orientation and film roughness must be further investigated and controlled. From the viewpoint of fundamental studies, the possibility of fabricating nearly monodisperse clusters of a wide range of sizes and densities will enable us to investigate many phenomena involving the magnetic behavior of materials, especially for clusters with a few nanometers in size as building blocks. Research on the magnetic properties of cluster-assembled materials is in its infancy and thus is fertile ground for both fundamental studies and applications.

\section{NON-EPITAXIAL ORIENTED $L 1_{0}$ FePt: $X\left(X=A g, C, B_{2} O_{3}\right)$ NANOCOMPOSITE FILMS}

\subsection{Non-Epitaxial Growth of (001) Textured $L 1_{0}$ FePt:X Films}

Under normal growth conditions, $L 1_{0}$-phase FePt and FePt based films often possess (111) preferred or random orientations. In order to control crystal orientation, the epitaxial growth of FePt film is needed to obtain the oriented films. The most common methods to obtain the $\mathrm{c}$ axis normal to the film plane $[(001)$ texture] are to use seed or buffer layers between a single-crystal substrate, such as $\mathrm{MgO}$, and the FePt and FePt-based films. Recently, highly $(001)$ textured $L 1_{0}$ FePt $[52,53]$ and FePt based films [54] have been obtained by a nonepitaxial growth method. Van et al. obtained (001) textured FePt and FePt based films by using multilayer deposition plus post annealing. It was found that orientations of FePt grains are affected by many preparation parameters, such as initial as-deposited film structure, composition, annealing time, and so on $[55,56]$.

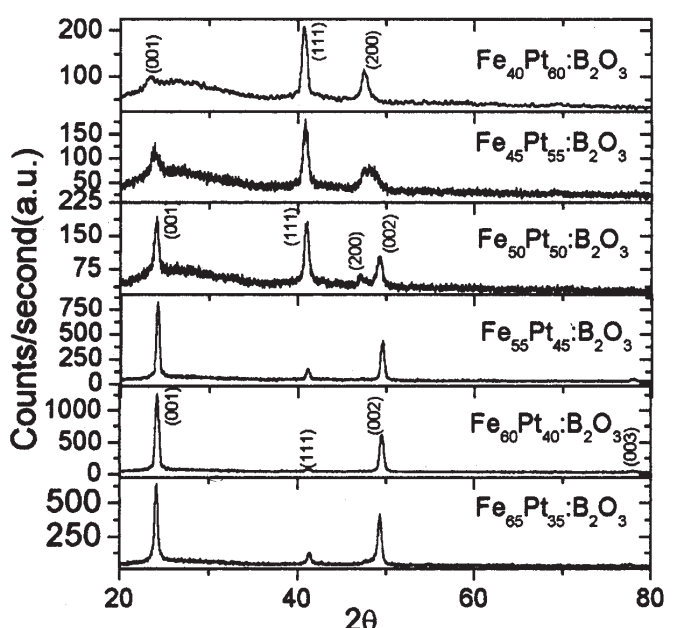

Figure 32. XRD patterns of the $\mathrm{Fe}_{\mathrm{x}} \mathrm{Pt}_{1-\mathrm{x}}: \mathrm{B}_{2} \mathrm{O}_{3}(11$ vol. \%) nanocomposite films. Films deposited directly on glass substrate and annealed at $550{ }^{\circ} \mathrm{C}$ for 30 minutes. Film thickness is $15 \mathrm{~nm}$.

Figure 32 shows the investigation of $\mathrm{Fe}$ concentration on orientation of $L 1_{0} \mathrm{FePt}: \mathrm{B}_{2} \mathrm{O}_{3}$ nanocomposite films. In the $\mathrm{Fe}-40$ at.\% film, XRD pattern shows (111), (200) peaks and relatively weak peak of (001). In the $\mathrm{Fe}-45 \mathrm{at} . \%$ film, relative intensity of (001) begins to increase and intensity of (111) and (200) decreases. The (200) and (002) peaks merge into one because of the close intensity and position of (200) and (002) peaks. This means the film is partially ordered. As Fe concentration further increases, the relative intensity of (111) peak decreases further. In Fe-60at.\% film, the (001) peak became predominant. The (111) peak is restrained and the (200) disappears. The high intensity ratio of $\mathrm{I}_{(001)} / \mathrm{I}_{(111)}$ indicates that (001) 
texture is favored and the $\mathrm{c}$ axis prefers to orient perpendicularly to the film plane in slightly Fe-rich films.

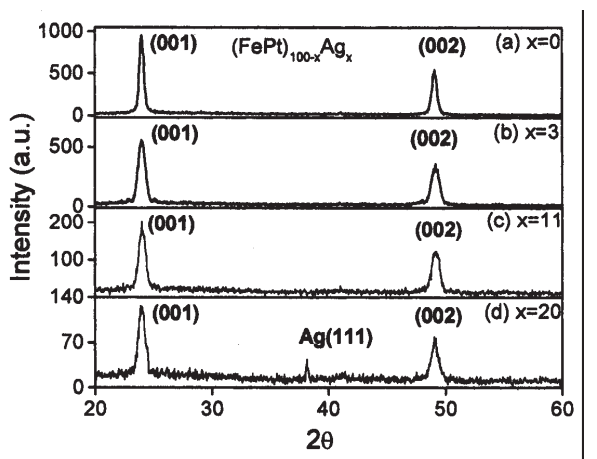

Figure 33. XRD patterns of FePt:Ag films with different Ag content. Films deposited directly on $\mathrm{Si}$ wafer and annealed at $600^{\circ} \mathrm{C}$ for 10 minutes. Film thickness is $10 \mathrm{~nm}$.

The effect of matrix concentration on orientation, intergranular interactions and magnetic properties has been investigated for $L 1_{0} \mathrm{FePt}: \mathrm{Ag}$ nanocomposite films. The Ag contents varied from 0 to $20 \mathrm{vol} \%$. As shown in Fig. 33, all films clearly show the (001) superlattice peaks indicating that the Ag content (matrix) has relatively small affection on orientation.

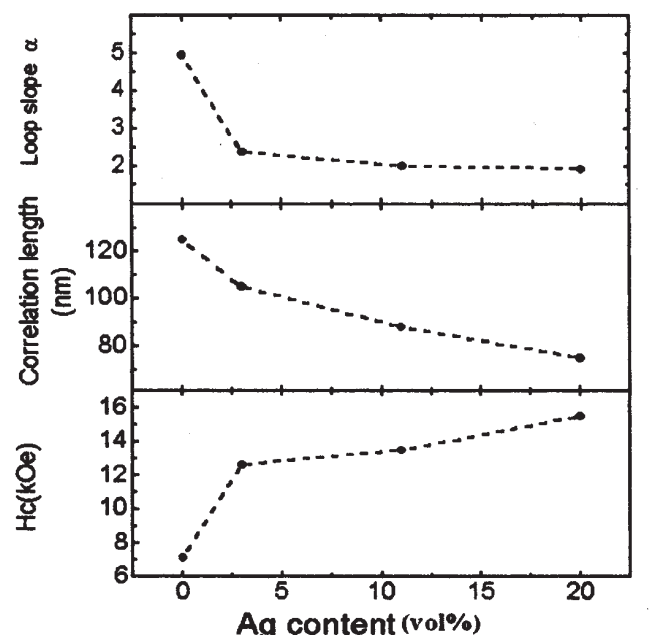

Figure 34. Hysteresis loop slope, correlation length and coercivity for various Ag contents (vol \%).

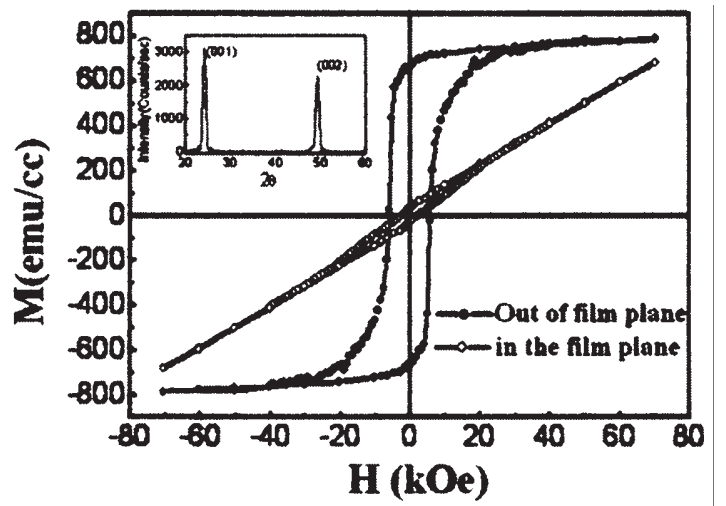

Figure 35. Hysteresis loop of FePt:C film. Film deposited directly on Si wafer and annealed at $550{ }^{\circ} \mathrm{C}$ for 5 minutes. Film thickness is $16 \mathrm{~nm}$. Inset is the XRD pattern of this sample.

A key aspect of the magnetism of nanocomposite films is the relation between intergranular interactions, the hysteresis-loop shape, and spatial correlations. Figure 34 shows the hysteresis loop slope, correlation length and coercivity of the FePt:Ag nanocomposite films with various Ag contents. Average interaction-domain sizes (correlation lengths) were deduced from MFM images. The coercivities and the hysteresis-loop slope parameter $\alpha=4 \pi \mathrm{d} M / \mathrm{d} H\left(H_{\mathrm{c}}\right)$ were determined from the hysteresis loops. With increasing Ag content the hysteresis-loop slope and the correlation length decrease, whereas the coercivity increases. The Ag increases the separation between the particles, thereby effectively exchange decoupling the grains.

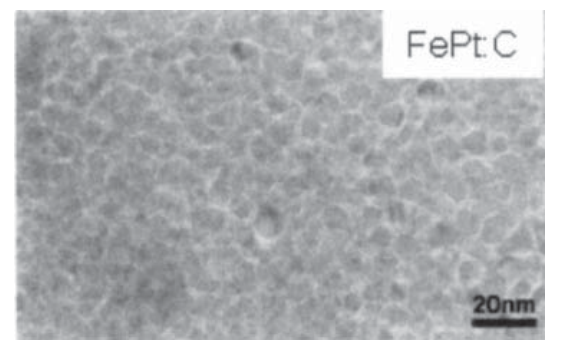

Figure 36. Bright-field TEM image of FePt:C film.

Hysteresis loops for $L 1_{0}$ nanocomposite FePt:C film is shown in Fig. 35. Inset is the XRD patterns of the same FePt:C film, on which (001), 
(002) peaks appear indicating that FePt grains are (001) oriented. The full width at half maximum (FWHM), obtained from the rocking curve of (001) peak, is $1.68^{\circ}$, conforming a high degree of (001) texturing. The loop shows perpendicular anisotropy with square shape in perpendicular direction due to the enhanced preferential FePt $L 1_{0}(001)$ texture. The perpendicular loop shows large coercivity $\left(H_{\mathrm{c}}=6.2 \mathrm{kOe}\right)$ and high remanence ratio $(S=0.9)$.

The nanostructure of nonepitaxially grown $L 1_{0} \mathrm{FePt}: \mathrm{C}$ thin film was characterized by TEM. As shown in Fig. 36, the bright-field image reveals that FePt grains with uniform size less than $5 \mathrm{~nm}$ are embedded in the carbon matrix and appear to be well isolated.

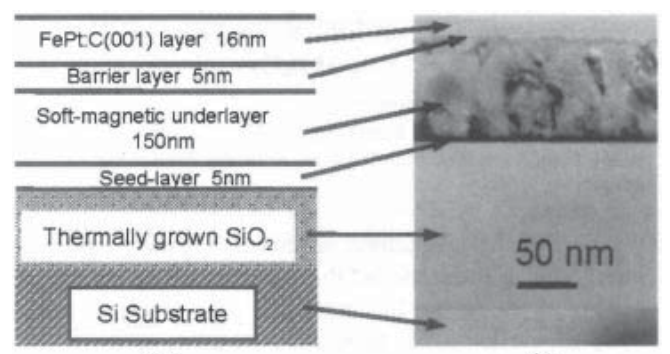

(a)

(b)

Figure 37. FePt:C double-layered perpendicular composite medium construction. (a) schematic sketch of the layers in the medium and (b) TEM cross-section image of this medium.

\subsection{Double-Layered $L 1_{0}$ FePt:C/FeCoNi Perpendicular Recording Media}

For practical applications, a nonepitaxially grown, double-layered thinfilm medium of nanocomposite FePt:C with a FeCoNi soft underlayer for high- density perpendicular magnetic recording was fabricated and investigated [56]. Figure 37(a) shows a schematic view of the cross section indicating substrate and each layer in the film. The FeCoNi soft underlayer (SUL) was first deposited on thermally oxidized Si substrates. The saturation magnetization flux density $\left(B_{\mathrm{s}}\right)$ for this layer is approximately $20 \mathrm{kG}$. The FePt:C layer was then deposited on the SUL at room temperature by a multilayer deposition technique and subsequent annealing. A $5 \mathrm{~nm}$ Ta barrier layer was introduced between SUL and FePt:C layer to reduce the diffusion between the SUL and FePt:C layer during the annealing process. A cross sectional TEM image, Fig. 37(b), verifies that this medium consists of nanocomposite FePt:C layer with FePt nanoparticles embedded in a $\mathrm{C}$ matrix and soft $\mathrm{FeCoNi}$ underlayer.
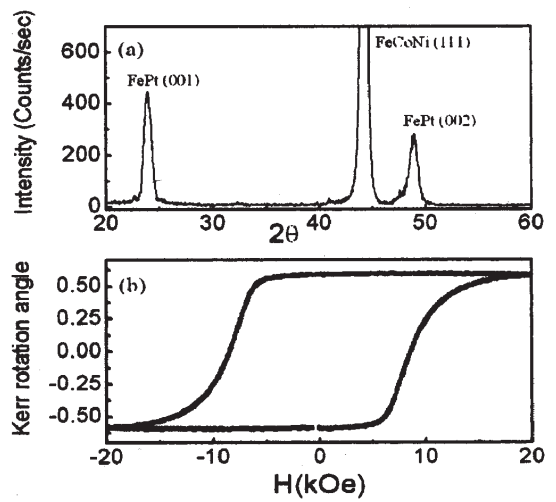

Figure 38. XRD pattern (a) and hysteresis loop (b) of FePt:C double-layered media. Loop measured by polar-Kerr Effect at room temperature.

Figure 38 shows the XRD pattern (a) and hysteresis loop (b) of FePt: $\mathrm{C}$ double-layered nanocomposite thin-film medium. The soft underlayer FeCoNi (111) peak and the $L 1_{0}$ FePt (001) and (002) peaks are shown only in the XRD pattern. This means that the preferred crystal orientation of $L 1_{0} \mathrm{FePt}: \mathrm{C}$ nanocomposite film is successfully obtained on this SUL by nonepitaxial growth. The polar-Kerr measurement shows a square loop that is only sensitive to the top layer; the Kerr effect data shown in this loop give the coercivity $H_{\mathrm{c}}=8.5 \mathrm{kOe}$, nucleation field $H_{\mathrm{n}}=5.65 \mathrm{kOe}$, remanence ratio $S=1$, and loop slope (at $H_{\mathrm{c}}$ ) $\alpha=3.3$, respectively.

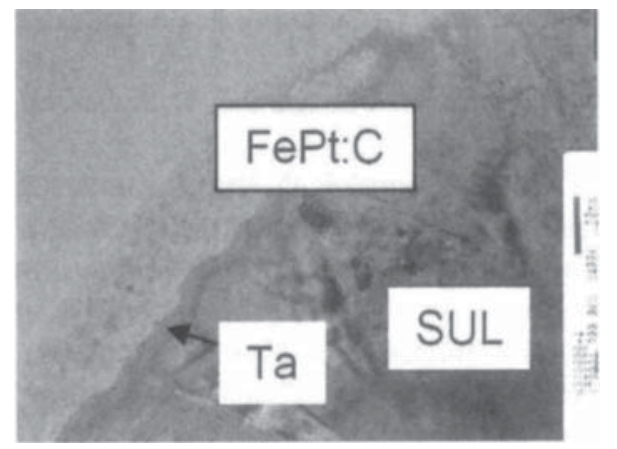

Figure 39. Cross-section TEM image of FePt:C double-layered media (scale bar: $20 \mathrm{~nm}$ ). 
The nanostructure of the double-layered nanocomposite FePt:C thin film medium was characterized by TEM. Electron diffraction shows that the crystallites are FePt with the $L 1_{0}$ structure. Figure 39 shows the brightfield and cross-section TEM image, which verifies that FePt crystallites are imbedded in $\mathrm{C}$ matrix and well isolated from each other.

These results suggest that nonepitaxially grown, (001) textured double- layered nanocomposite $L 1_{0} \mathrm{Fe}(\mathrm{Co}) \mathrm{Pt}$-based films with perpendicular anisotropy are a promising candidate to realize extremely high-density perpendicular recording.

\section{Acknowledgements}

The authors are grateful to many colleagues for assistance and helpful discussions, and to NSF-MRSEC, INSIC, DOE, ARO, and CMRA for financial support.

\section{References}

[1] H. Haberland (Ed.), "Clusters of Atoms and Molecules," Springer-Verlag, 1994.

[2] W. Ekardt (Ed.), "Metal Clusters," Whily 1999.

[3] K.-H. Meiwes-Broer (Ed.), "Metal Cluster at Surfaces," Springer 2000.

[4] J. P. Bucher, D. C. Douglass, and L.A. Bloomfield, Phys. Rev. Lett. 66, 3052 (1991).

[5] I. M. L. Billas, J. A. Becker, A. Châtelain, and W. A. de Heer, Phys. Rev. Lett. 71, 4067 (1993).

[6] I. M. L. Billas, A. Châtelain, and W. A. de Heer, Science 265, 1682 (1994).

[7] I. M. L. Billas, W. A. de Heer, and A. Châtelain, J. Non-Cryst. Solids 179, 316 (1994).

[8] A. J. Cox, J. G. Louderback, S. E. Apsel, and L.A. Bloomfield, Phys. Rev. B 49 12295 (1994).

[9] S. E. Apsel, J. W. Emmert J. Deng, and L. A. Bloomfield, Phys. Rev. Lett. 76, 1441 (1996).

[10] K. W. Edmonds, C. Binns, S. H. Baker, S. C. Thornton, and C. Norris, Phys. Rev. B 60, 472 (1999).

[11] S. Stavroyiannis, I. Panagiotopoulos, D. Niarchos, J. A. Christodoulides, Y. Zhang, and G. C. Hadjipanayis, Appl. Phys. Lett. 73, 3453 (1998).

[12] M. Vu, Y. Liu, A. Moser, D. Weller, and D. J. Sellmyer, Appl. Phys. Lett. 75, 3992 (1999).
[13] B. Bian, K. Sato, and Y. Hirotsu, Appl. Phys. Lett. 75, 3686 (1999).

[14] T. Suzuki, T. Kiya, N. Honda, and K. Ouchi, J. Magn. Magn. Mater. 235, 312 (2001).

[15] T. Vang, E. Ahmad, and T. Suzuki, J. Appl. Phys. 91, 6860 (2002).

[16] R. Wood, IEEE Trans. Magn. 36, 36 (2000).

[17] R. A. McCurrie and P. Gaunt, Philos. Mag. 13, 567 (1966).

[18] B. Zhang and W. A. Soffa, IEEE Trans. Magn. 26, 1388 (1990).

[19] E. E. Fullerton, C. H. Sowers, J. E. Pearson, S. D. Bader, J. B. Patel, and X. Z. Wu, J. Appl. Phys. 81,5937 (1997)

[20] S. Sun, C. B. Murray, D. Weller, L. Folks, and A. Moser, Science 287,1989 (2000).

[21] H. Zeng, J. Li, J. P. Liu, Z. L. Wang, and S. Sun, Nature 420, 395 (2002).

[22] H. Haberland, M. Karrais, M. Mall and Y. Thumer, J. Vac. Sci. Technol. A 10, 3266 (1992).

[23] K. Sattler, J. Muhlbach, and E. Recknagel, Phys. Rev. Lett. 45, 821 (1980).

[24] S. S. Riley, E. Parks, C. Mao, L. Pobo, and S. J. Wexler, Phys. Chem. 86, 3911 (1982).

[25] F. Frank, W. Schulze, B. Tesche, J. Urban, and B. Winter, Surf. Sci. 156, 90 (1985).

[26] H. Schaber, T. P. Martin, Surf. Sci. 156, 64 (1985).

[27] S. H. Baker, S. C. Thornton, A. M. Keen, T. I. Preston, C. Norris, K. W. Edmonds, and C. Binns, Rev. Sci. Instrum. 68, 1853 (1997).

[28] S. H. Baker, S. C. Thornton, K. W. Edmonds, M. J. Maher; C. Norris, and C. Binns, Rev. Sci. Instrum. 71, 3178 (2000).

[29] H. Haberland, M. Mall, M. Moseler, Y. Qiang, T. Reiners, and Y. Thurner, J. Vac. Sci. Technol. A 12, 2925 (1994).

[30] See: http://www.oareserch.co.uk/ and http://www.mantisdeposition.com/.

[31] M. D. Upward, B. N. Cotier, P. Moriarty, P. H. Beton, S. H. Baker, C. Binns and Edmonds, J. Vac. Sci. Technol. B 18, 2646 (2000).

[32] J. Söderlund, L. B. Kiss, G. A. Niklasson, and C. G. Granqvist, Phys. Rev. Lett. 80, 2386 (1998).

[33] K. W. Edmonds, C. Binns, S. H. Baker, S. C. Thornton, and C. Norris, Phys. Rev. B 60, 472 (1999).

[34] C. Binns, M. J. Maher, Q. A., Pankhurst, D. Kechrakos, and K. N. Trohidou, Phys, Rev. B 66, 184413 (2002).

[35] Y. Qiang, R. F. Sabiryanov, S. S. Jaswal, Y. Liu, H. Haberland and D. J. Sellmyer, Phys. Rev. B 66, 064404 (2002).

[36] T. Hihara and K. Sumiyama, J. Appl. Phys. 84, 5270 (1998).

[37] D. L. Peng, K. Sumiyama, S. Yamamuro, T. Hihara, and T. J. Konno, Appl. Phys. 
Lett. 74, 76 (1999).

[38] D. L. Peng, K. Sumiyama, T. J. Konno, T. Hihara, S. Yamamuro, Phys. Rev. B 60, 2093 (1999).

[39] D. L. Peng, T. Hihara, K. Sumiyama, and H. Morikawa, J. Appl. Phys. 92, 3075 (2002).

[40] T. J. Konno, S. Yamamuro, and K. Sumiyama, J. Vac. Sci. Technol. B 20, 834 (2002).

[41] T. J. Konno, S. Yamamuro, and K. Sumiyama, J. Appl. Phys. 90, 834 (2001).

[42] F. R. de Boer, R. Boom, W. C. M. Mattens, A. R. Miedema, and A. K. Niessen, in "Cohesion in Metals Transition Metal Alloys," North-Holland, Amsterdam 1988.

[43] Y. Xu, Z. G. Sun, Y. Qiang, and D. J. Sellmyer, J. Appl. Phys. 93, 8289 (2003).

[44] Y. Xu, Z. G. Sun, Y. Qiang, and D. J. Sellmyer, J. Magn. Magn. Mater. 266, 164 (2003).

[45] Y. Xu, M. L. Yan, J. Zhou, and D. J. Sellmyer, J. Appl. Phys. 97, 10J320 (2005).

[46] B. Rellinghaus, S. Stappert, M. Acet, and E. F. Wassermann, J. Magn. Magn. Mater. 266, 142 (2003).

[47] S. Stappert, B. Rellinghaus, M. Acet, and E. F. Wassermann, J. Cryst. Grow. 252, 440 (2003).

[48] Y. Xu, M. L. Yan, and D. J. Sellmyer, IEEE Trans. Magn. 40, 2525 (2004).

[49] D. J. Sellmyer, C. P. Luo, M. L. Yan, and Y. Liu, IEEE Trans. Magn. 37, 1286 (2001).

[50] M. P. Sharrock, J. Appl. Phys. 76, 6413 (1994).

[51] Y. Xu, Z. S. Shan, J. P. Wang, and C. T. Chong, J. Magn. Magn. Mater. 232, 103 (2001).

[52] M. L. Yan, N. Powers, and D. J. Sellmyer, J. Appl. Phys. 93, 8292 (2003).

[53] H. Zeng, M. L. Yan, N. Powers, and D. J. Sellmyer, Appl. Phys. Lett. 80, 2350 (2002).

[54] M. L. Yan, H. Zeng, N. Powers, and D. J. Sellmyer, J. Appl. Phys. 91, 8471 (2002).

[55] Y. Shao, M. L. Yan, and D. J. Sellmyer, J. Appl. Phys. 93, 8152 (2003).

[56] M. L. Yan, X. Z. Li, L. Gao, S. H. Liu, D. J. Sellmyer, R. J. M. van de Veerdonk, and K. W. Wierman, Appl. Phys. Lett. 83, 3332 (2003). 